
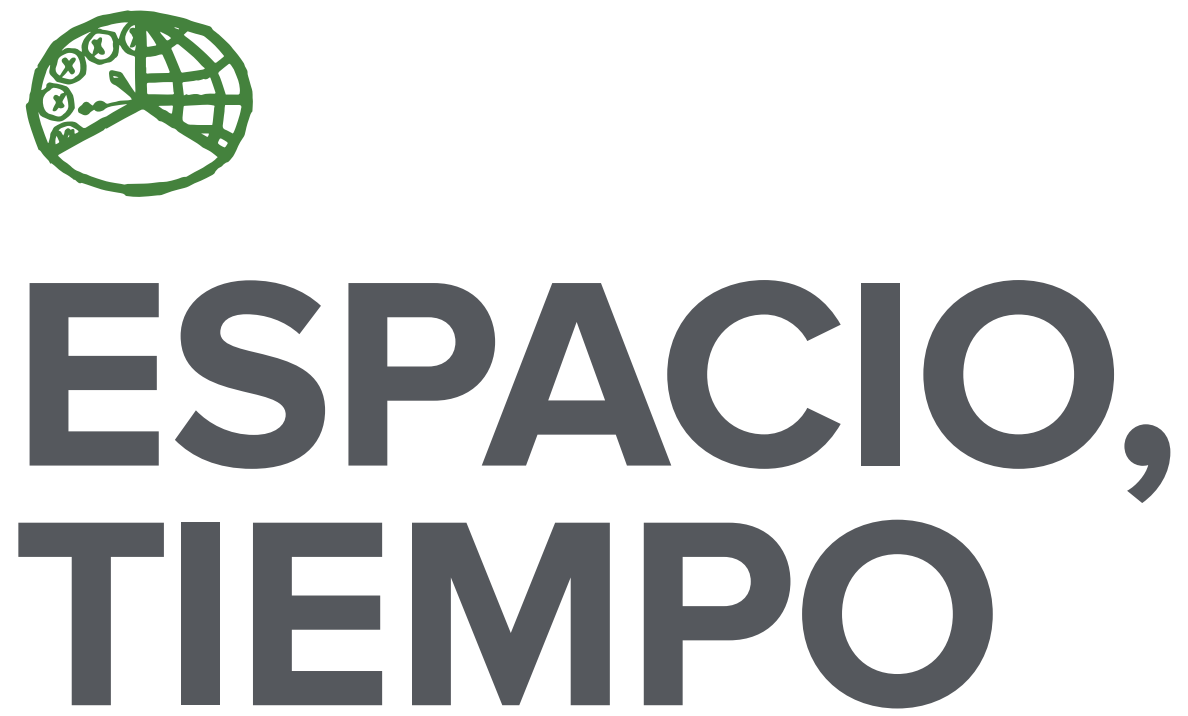

AÑOS 2015-2016

ISSN 1130-2968

E-ISSN 2340-146X
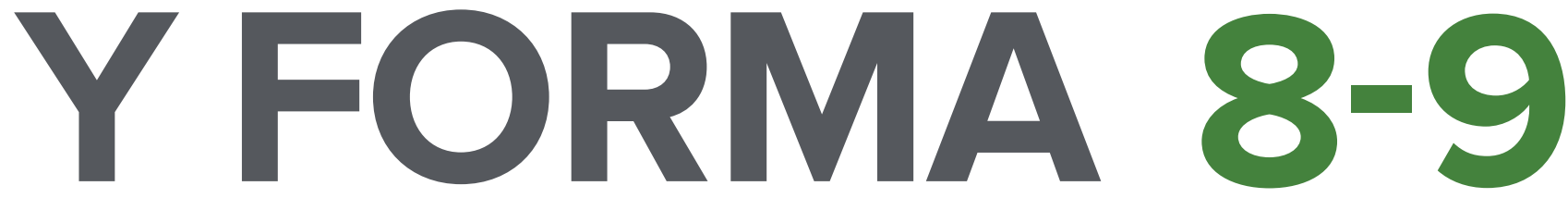

SERIE VI GEOGRAFÍA

REVISTA DE LA FACULTAD DE GEOGRAFÍA E HISTORIA 

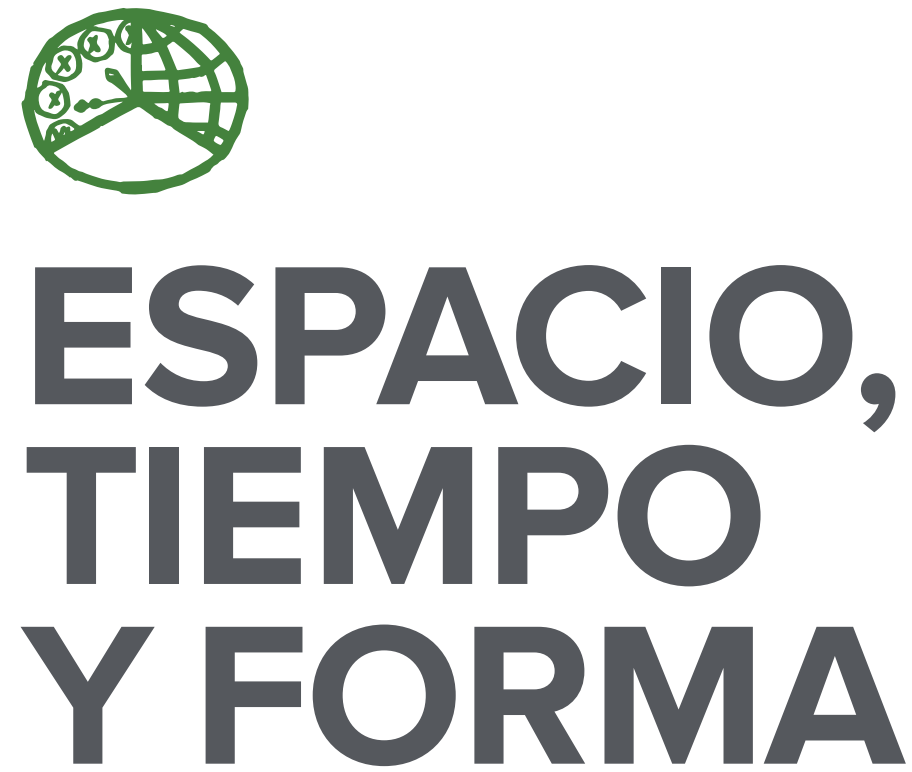

AÑOS 2015-2016

ISSN 1130-2968

E-ISSN 2340-146X

SERIE VI GEOGRAFÍA

REVISTA DE LA FACULTAD DE GEOGRAFÍA E HISTORIA

DOI: http://dx.doi.org/10.5944/etfvi.8-9.2016

\section{UกED}

UNIVERSIDAD NACIONAL DE EDUCACIÓN A DISTANCIA 
La revista Espacio, Tiempo y Forma (siglas recomendadas: ETF), de la Facultad de Geografía e Historia de la UNED, que inició su publicación el año 1988, está organizada de la siguiente forma:

$$
\begin{aligned}
& \text { SERIE I - Prehistoria y Arqueología } \\
& \text { SERIE II - Historia Antigua } \\
& \text { SERIE III - Historia Medieval } \\
& \text { SERIE IV - Historia Moderna } \\
& \text { SERIE V - Historia Contemporánea } \\
& \text { SERIE VI - Geografía } \\
& \text { SERIE VII - Historia del Arte }
\end{aligned}
$$

Excepcionalmente, algunos volúmenes del año 1988 atienden a la siguiente numeración:

$$
\begin{aligned}
& \mathrm{N}^{\circ} 1 \text { - Historia Contemporánea } \\
& \mathrm{N}^{\circ} 2 \text { - Historia del Arte } \\
& \text { N. }{ }^{\circ} 3 \text { - Geografía } \\
& \mathrm{N} .^{\circ} 4 \text { - Historia Moderna }
\end{aligned}
$$

ETF no se solidariza necesariamente con las opiniones expresadas por los autores.

Espacio, Tiempo y Forma, Serie vi está registrada e indexada, entre otros, por los siguientes Repertorios Bibliográficos y Bases de Datos: DICE, ISOC (CINDOC), RESH, IN-RECH, Dialnet, e-sPacio, UNED, CIRC, MIAR, FRANCIS, PIO, ULRICH'S, SUDOC, 2DB, ERIH (ESF).

\author{
UNIVERSIDAD NACIONAL DE EDUCACIÓN A DISTANCIA \\ Madrid, 2015-2016 \\ SERIE VI · GEOGRAFÍA N. ${ }^{\circ} 8-9,2015-2016$ \\ ISSN 1130-2968 · E-ISSN 2340-146x \\ DEPÓSITO LEGAL \\ M-21.037-1988 \\ URL \\ ETF VI · GEOGRAFÍA $\cdot$ http://revistas.uned.es/index.php/ETFVI \\ COMPOSICIÓN \\ Carmen Chincoa Gallardo · http://www.laurisilva.net/cch
}

Impreso en España · Printed in Spain

(c) (7) (8) Esta obra está bajo una licencia Creative Commons

Reconocimiento-NoComercial 4.0 Internacional. 


\title{
LA INCORPORACIÓN DE LOS PORTAFOLIOS ELECTRÓNICOS EN EL PROCESO ENSEÑANZA-APRENDIZAJE Y EVALUACIÓN DE LA GEOGRAFÍA EN EL ALUMNADO DE EDUCACIÓN A DISTANCIA
}

\section{THE INCORPORATION OF ELECTRONIC PORTFOLIOS LEARNING IN GEOGRAPHY AND EVALUATION PROCESS IN DISTANCE EDUCATION STUDENTS}

\author{
Francisco José Morales Yago ${ }^{1}$ \\ Recepción: 11/05/2014 · Aceptación: 19/12/2014 \\ DOI: http://dx.doi.org/10.5944/etfvi.8-9.2016.1636
}

\begin{abstract}
Resumen
El presente artículo expone el sentido y las características de un portfolio electrónico aplicado en un contexto universitario de enseñanza a distancia, en la asignatura: «Introducción a la investigación en Espacios Sociales» correspondiente al Máster de la UNED denominado: «Métodos y técnicas avanzadas de investigación histórica, artística y geográfica», dentro del itinerario de Geografía. La plataforma ALF de la UNED será el instrumento de e-Learning que permitirá impartir formación, gestionar y compartir documentos, crear y participar en comunidades temáticas, así como realizar proyectos online. A través de la misma se llevará a cabo todo el intercambio de documentación, aportación de ideas, orientaciones básicas e incluso el seguimiento de los procesos desde el inicio hasta su finalización.
\end{abstract}

Palabras clave

Porfolio; enseñanza-aprendizaje; innovación educativa; evaluación formativa; autoevaluación; aprendizaje reflexivo.

\section{Abstract}

This communication presents the meaning and characteristics of an electronic portfolio applied in a university context of distance learning in the course: «Introduction to Research in Social Spaces» for the Master of UNED called: «Methods and advanced techniques historical, artistic and geographical research «within

1. UNED. Grupo de investigación «CULTURPAIS». Departamento de Geografía. Facultad de Geografía e Historia. C/Senda del Rey n 7. 28040. Madrid (España).<fjmorales@geo.uned.es>. 
the itinerary of Geography. The ALF platform UNED will be e-Learning tool that will allow train, manage and share documents, create and participate in thematic communities, as well as make online projects. Through the same will take place around the exchange of documentation, brainstorming, basic orientations and even tracking processes from start to finish.

\section{Keywords}

Portfolio; teaching and learning; educational innovation; formative assessment; self-evaluation; reflective learning. 


\section{INTRODUCCIÓN}

El portfolio presentado pone su énfasis en el seguimiento de los trabajos de los estudiantes basándose en el progreso continuado de las competencias profesionales que se han desarrollado a lo largo de los estudios de Geografía y que se resumen en esta asignatura a través de su carácter práctico, ya que se deberá llevar a cabo un trabajo basado en la elaboración personal de datos y aplicación práctica de los conocimientos adquiridos en las diversas fuentes consultadas. Para ello será necesario adquirir previamente un conocimiento teórico de las fuentes documentales más utilizadas en el estudio de los procesos demográficos y en las actividades de los sectores de producción primario, secundario y terciario. La profundidad de conocimientos en alguno de esos sectores vendrá marcada por el tema de investigación seleccionado; no obstante en todo trabajo geográfico será imprescindible acudir también al estudio teórico previo de las fuentes cartográficas como fuente de información, representación y apoyo de las fuentes documentales para conseguir la máxima expresividad en el documento final.

El avance en la adquisición de este proceso de enseña-aprendizaje se basará en el desarrollo de las competencias mediante la publicación de evidencias de logro (rúbricas) que documentarán el nivel de progreso de las citadas competencias. El portfolio electrónico planteado tendrá carácter individual y estará sostenido por un mecanismo de apoyo por parte del profesor de la asignatura, mediante el que aportará retroalimentación continuada a los estudiantes, lo que les facilitará el ajuste de sus actuaciones a las competencias profesionales planteadas.

El objetivo principal que nos mueve a experimentar con el portfolio como procedimiento de evaluación y aprendizaje, es la aspiración que tenemos de convertir a nuestros alumnos en estudiantes reflexivos y críticos. De ello se desprende que en nuestro programa formativo, hemos priorizado entre otras, competencias tales como la capacidad de reflexión y análisis crítico. Pensamos que ambas juegan un papel fundamental para nuestros estudiantes como futuros profesionales de las Ciencias Sociales, y en concreto de la Geografía como ciencia que estudia la actividad humana y su impacto en el paisaje además de otras cuestiones derivadas de los usos del suelo. Las evidencias demuestran que el uso de portfolios promueve el desarrollo de las mismas, y además nos permite obtener un mayor control del trabajo realizado por el alumnado, cuestión que toma una especial relevancia dentro del crédito europeo iniciado hace pocos años tras la puesta en funcionamiento del Plan Bolonia. Además de estas razones, encontramos muchas más, tanto teóricas como prácticas, que justifican y aconsejan el uso del portfolios en diversidad de contextos educativos y con distintos propósitos. Desde una visión más metodológica y técnica, podemos destacar la insatisfacción que existe derivada de la utilización de metodologías basadas en enfoques cuantitativos que derivan en el abuso de pruebas memorísticas finales con la mera consideración de los resultados.

El Portfolio presenta aspectos interesantes como la posibilidad de realizar un trabajo acumulativo, secuenciado y ordenado; también otorga al alumnado un importante protagonismo que le ayudará en el proceso de reflexión y mejora. Finalmente 
se pretende un aprendizaje no meramente descriptivo, sino razonado de tal forma que se pueda mantener a lo largo del tiempo y permita una aplicación razonada en futuros procesos de aprendizaje e investigación por parte del alumnado implicado.

\section{SECUENCIACIÓN DEL PORTFOLIO}

La asignatura se imparte a lo largo de cuatro meses, de mediados de febrero a mediados de junio. Al objeto de que la distribución de las tareas a realizar se programe de forma ordenada y gradual se sugiere la siguiente secuencia temporal:

\begin{tabular}{|c|c|c|c|}
\hline $1 / 2$ Febrero a $1 / 2$ Marzo & $1 / 2$ Marzo a $1 / 2$ abril & $1 / 2$ abril a $1 / 2$ Mayo & $1 / 2$ abril a $1 / 2$ Mayo \\
\hline $\begin{array}{l}\text { Estudio de las fuentes } \\
\text { documentales y } \\
\text { cartográficas y } \\
\text { determinación del } \\
\text { tema objeto de estudio, } \\
\text { definiendo la metodología } \\
\text { a seguir. }\end{array}$ & $\begin{array}{l}\text { Selección, } \\
\text { localización, consulta } \\
\text { y toma de datos de las } \\
\text { fuentes directas. }\end{array}$ & $\begin{array}{l}\text { Elaboración de la } \\
\text { información de } \\
\text { todas las fuentes } \\
\text { consultadas. }\end{array}$ & $\begin{array}{l}\text { Ordenación, } \\
\text { redacción, } \\
\text { conclusiones finales, } \\
\text { puesta a punto y } \\
\text { entrega del trabajo } \\
\text { de investigación } \\
\text { para ser evaluado. }\end{array}$ \\
\hline
\end{tabular}

Consulta bibliográfica sobre trabajos análogos.

tabla 1. CROnograma de planificación. Fuente. Elaboración Propia.

La naturaleza del trabajo desarrollado, su individualidad y singularidad hacen muy difícil un problema extendido actualmente en los trabajos universitarios: El plagio. Llevar a cabo una falsificación será complicado ya que el profesor que dirige este trabajo asistirá al progreso del alumno desde el comienzo, corrigiendo fallos, mejorando estilo, e incluso solicitando documentos acreditativos que verifiquen los contenidos expuestos. En el caso de la asignatura de Máster que impartimos cada alumno deberá elegir una ciudad de tipo medio para investigar sobre las repercusiones del envejecimiento poblacional en el espacio urbano, estudiando las dinámicas de envejecimiento y su repercusión sobre ese espacio utilizando metodologías y fuentes de investigación apropiadas a la naturaleza de este tipo de trabajos.

El alumno deberá aprender a seleccionar información precisa y significativa, analizarla y extraer las conclusiones oportunas. Por todo ello deberá construir pirámides de población general y por barrios o distritos, analizar los perfiles de las mismas y extraer conclusiones sobre tasas de mortalidad, fecundidad y crecimiento de la población. A continuación utilizará una serie de indicadores significativos como el análisis de la población de más de 65 años por sexo, nivel de instrucción y profesiones desempeñadas en cada uno de los barrios o distritos; el análisis del régimen de tenencia de las viviendas (en propiedad, alquiler o cesión); el estudio del número de personas que ocupan la vivienda y edad de las mismas, nivel de autonomía personal (total, parcial o absoluta) o forma de resolución en caso exista alguna dependencia (centros públicos, privados o concertados). 
Otro importante apartado del trabajo consistirá en la realización de un mapa de equipamientos y servicios así como su accesibilidad en los distintos barrios de la ciudad en donde entre otros figuren equipamientos tales como los centros culturales y educativos por niveles (bibliotecas, universidad Senior, universidad y centros de cultura popular, etc.); servicios sanitarios (ambulatorios, dispensarios, hospitales con servicio geriátrico, etc.); Instalaciones para el ocio, centros de día (señalando la titularidad: pública, privada o concertada), Servicios comerciales (farmacias, bancos, etc.), residencias para personas mayores instaladas en el conjunto urbano (indicando la titularidad: pública, privada o concertada y el coste económico de la plaza), mapa de transportes colectivos y su adaptación a las personas mayores, estado actual de la existencia de barreras arquitectónicas, estado actual del mobiliario urbano indicando el estado de conservación de aseos públicos, bancos en zonas ajardinadas, o índices de dotación para mayores de 65 años en los distintos barrios de la ciudad.

La Geografía es la ciencia del análisis científico del paisaje, por tanto no podía faltar en este trabajo un apartado, antes de llegar a las conclusiones y propuestas de mejora, dedicado a la huella poblacional en el paisaje urbano, por ello se trabajará los grados de renovación de las edificaciones en los barrios, rehabilitación de las edificaciones, intervención de las administraciones públicas en la conservación del patrimonio residencial y urbanístico, finalizando con la elaboración de un plano del barrio estudiado en donde se reflejen las principales actuaciones urbanísticas para la renovación y rehabilitación del mismo en las últimas décadas, pudiendo realizar para el análisis cualitativo una encuesta sobre el grado de satisfacción ciudadana por barrios y propuestas de mejora para una mayor calidad de vida de las personas mayores.

\section{CONCLUSIONES}

Como hemos comentado con anterioridad, el uso del portafolios en la materia «Investigación en Espacios Sociales», surge bajo la necesidad de implantar e innovar metodologías de enseñanza y evaluación de cara a la convergencia del sistema de educación superior español al ámbito europeo. No cabe duda de que la implantación de este tipo de experiencias va precedida y seguida de un proceso de reflexión y análisis sobre los nuevos modelos pedagógicos y su implicación práctica en el contexto de las aulas universitarias. La primera reflexión importante a tener en cuenta, es que aunque desde la nueva perspectiva del crédito europeo, se establece el énfasis en el trabajo del alumno, el trabajo del docente no pasa a estar en un segundo plano, todo lo contrario, la planificación y conocimientos metodológicos así como el domino pedagógico de una serie de pautas didácticas por parte del profesor serán más exigentes dentro de esta nueva perspectiva, es por ello que el profesor deberá estar preparado y formado pedagógicamente, puesto que es un punto esencial para que el cambio se produzca con éxito. 


\section{BIBLIOGRAFÍA}

Agra, M.J. (2003) El portafolio como herramienta de análisis en experiencias de formación on line y presenciales. En Enseñanza: Anuario Interuniversitario de didáctica, núm.2I, págs. IOI-II4

BARBERÀ, E. (2006). Evaluación por portafolio en la Universidad. Forum sobre docencia Universitaria de la Universidad de Barcelona. Extraído el I-02-2015, de $<$ <ttp://www.ub.edu/forum/Catalan/welcome.htm>

BARBERÀ, E. (2008). El estilo e-portafolio. Barcelona, Editorial UOC.

BARRAGÁN, R. (2005). El portafolio, metodología de evaluación y aprendizaje de cara al nuevo espacio Europeo de Educación superior. Una experiencia práctica en la Universidad de Sevilla. Revista Latinoamericana de Tecnología Educativa, 4 (I), I2I--I39. Disponible el 27/03/2015 en: <http://www.unex.es/didactica/RELATEC/sumario_4_I.htm>

Consejo de Europa (2004). Portafolio Europeo de Lenguas. Niveles Europeos de autoevaluación de la capacidad lingüística. Disponible el 5/03/2015 en $<$ http://www.coe.int/portfolio>

Corominas, E. (2000). ¿Entramos en la era portafolios? Bordón, 52(4), 509- 52I.

De Miguel, M. (Dir.) (2005). Modalidades desde enseñanza centradas en el desarrollo de competencias. Orientaciones para promover el cambio metodológico en el marco del EEES. Oviedo: Ediciones de la Universidad de Oviedo. Disponible el 20/02/2015 en $<$ http://www.uned.es/educacion> [EDUDoc]

Gallego, D; Cacheiro, M. L; Martín, A; Angel, W. El Eporfolio como estrategia de enseñanza y aprendizaje» [artículo en línea]. EDUTEC, Revista Electrónica de Tecnología Educativa. Núm. 30/ Noviembre 2009. [Fecha de consulta: II/03/2015]. $<$ http://edutec.rediris.es/revelec2/revelec30/> ISSN II35-9250.

Hills, J.R. (198I). Evaluación y medición en la Escuela. México: Kapelusz

JONES, S. (2008). E---portfolios and how they can support Personalisation. Improving learning through technology. UK: Becta. Disponible el 3/I/2009 en $<$ http://events.becta.org.uk/content_files/corporate/resources/events/2007/jan/bett_007/ betto7_eportfolios_support_personalisation.pdf>

KLENOWSKI, V. (2007). Desarrollo de portafolios para el aprendizaje y la evaluación. Madrid: Narcea.

Medina, A. (2009) Formación y desarrollo de las competencias básicas. Madrid: Univérsitas.

Pérez, R. y Otros (20I2). El portfolio. Aprendizajes, competencias y evaluación. Madrid: UNED.

Sutherland, S (2005). ePortfolios: a personal learning space. S. Freitas, de y C. Yapp. Personalisation in the $2 I^{\text {st }}$ Century. Stafford: Network Press 


\begin{tabular}{|c|c|c|}
\hline OBJETIVOS & $\begin{array}{l}\text { COMPETENCIAS GENERALES DE } \\
\text { LA MATERIA INTRODUCCIÓN A LA } \\
\text { INVESTIGACIÓN ESPACIOS SOCIALES }\end{array}$ & $\begin{array}{l}\text { ACTIVIDADES } \\
\text { FORMATIVAS }\end{array}$ \\
\hline $\begin{array}{l}\text { - Conocer las principales fuentes } \\
\text { documentales y su aplicación } \\
\text { práctica que permitan al alumno } \\
\text { aproximarse a la realidad social } \\
\text { en distintos ámbitos españoles. } \\
\text { - Estimular la capacidad de } \\
\text { análisis de los fenómenos que } \\
\text { intervienen en el modelado de } \\
\text { los espacios sociales mediante } \\
\text { su observación, localización y } \\
\text { comparación. } \\
\text { - Desarrollar la actitud crítica } \\
\text { del alumno a través del manejo } \\
\text { y elaboración de datos de las } \\
\text { fuentes consultadas y de la } \\
\text { bibliografía específica para } \\
\text { atender los diferentes recursos } \\
\text { del análisis del espacio social. } \\
\text { - Adquirir los conocimientos y } \\
\text { destrezas imprescindibles para } \\
\text { la elaboración de un trabajo } \\
\text { de investigación sobre algún } \\
\text { fenómeno de interés social. }\end{array}$ & $\begin{array}{l}\text { - Poseer y comprender conocimientos } \\
\text { que aporten una base u oportunidad de } \\
\text { ser originales en el desarrollo } \\
\text { y/o aplicación de ideas, a menudo en un } \\
\text { contexto de investigación. } \\
\text { - Que los estudiantes sepan aplicar } \\
\text { los conocimientos adquiridos y su } \\
\text { capacidad de resolución de problemas } \\
\text { en entornos nuevos o poco conocidos } \\
\text { dentro de contextos más amplios (o } \\
\text { multidisciplinares) relacionados con su } \\
\text { área de estudio. } \\
\text { - Que los estudiantes sean capaces de } \\
\text { integrar conocimientos y enfrentarse } \\
\text { a la complejidad de formular juicios } \\
\text { a partir de una información que, } \\
\text { siendo incompleta o limitada, incluya } \\
\text { reflexiones sobre las responsabilidades } \\
\text { sociales y éticas vinculadas a la } \\
\text { aplicación de sus conocimientos y } \\
\text { juicios. } \\
\text { - Que los estudiantes sepan comunicar } \\
\text { sus conclusiones y los conocimientos } \\
\text { y razones últimas que las sustentan } \\
\text { a públicos especializados y no } \\
\text { especializados de un modo claro y sin } \\
\text { ambigüedades. } \\
\text { - Que los estudiantes posean las } \\
\text { habilidades de aprendizaje que les } \\
\text { permitan continuar estudiando de un } \\
\text { modo que habrá de ser en gran medida } \\
\text { autodirigido o autónomo. }\end{array}$ & $\begin{array}{l}\text { - Utilización fuentes } \\
\text { primarias } \\
\text { - Búsquedas web } \\
\text { - Manejo campus virtual } \\
\text { ALF } \\
\text { - Redacción de trabajo } \\
\text { - Diario de campo }\end{array}$ \\
\hline
\end{tabular}

TABLA N ${ }^{\circ}$ 2. OBJETIVOS Y COMPETENCIAS GENERALES DE LA MATERIA: INTRODUCCIÓN A LA INVESTIGACIÓN EN ESPACIOS SOCIALES Fuente: Elaboración Propia 


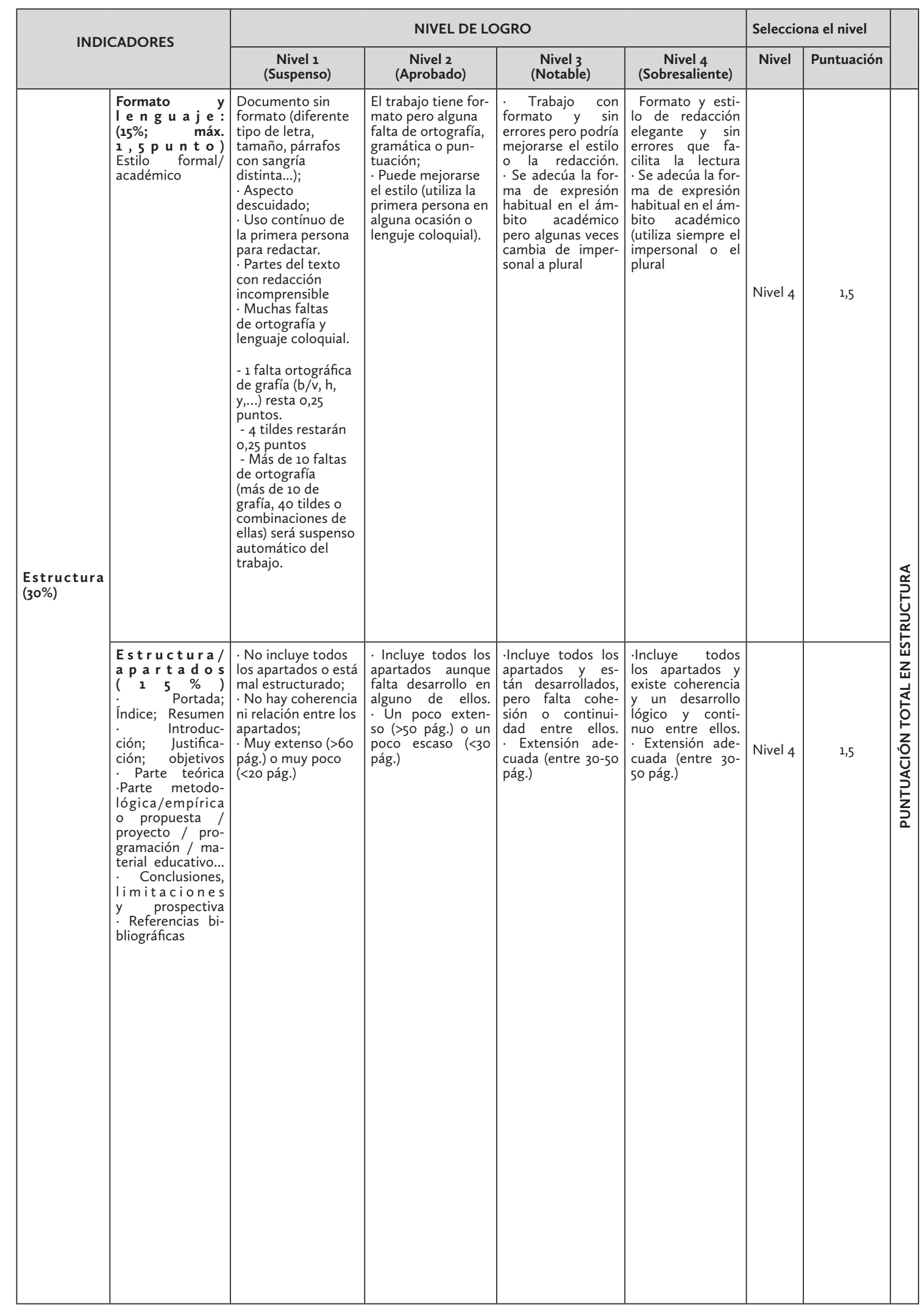




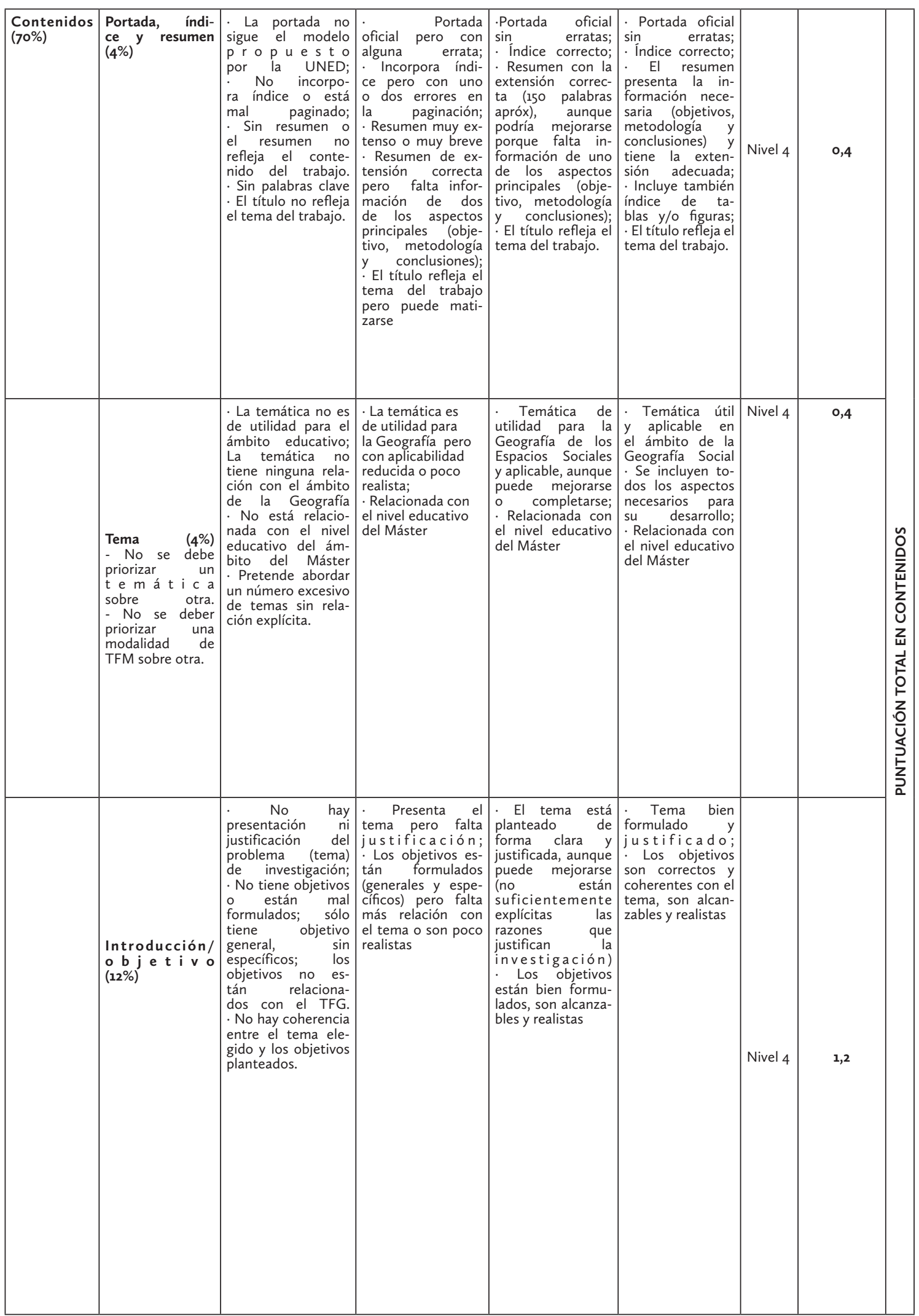




\begin{tabular}{|c|c|c|c|c|c|c|c|}
\hline $\begin{array}{l}\text { Marco Teórico } \\
(15 \%) \\
\text { - Detectar plagio } \\
\text { en un TFG será } \\
\text { motivo de sus- } \\
\text { penso. }\end{array}$ & $\begin{array}{l}\text { - No hay relación } \\
\text { con el problema (el } \\
\text { tema) y los objeti- } \\
\text { vos del TFM; } \\
\text { - No hay orden en } \\
\text { los contenidos; } \\
\text { - Faltan definiciones } \\
\text { de conceptos; } \\
\text { - Afirmaciones sin } \\
\text { justificar; } \\
\text { - Fuentes poco } \\
\text { fiables o pocas } \\
\text { fuentes consultadas } \\
\text { (<10); } \\
\text { - No hay fuentes } \\
\text { primarias; } \\
\text { - Hay textos } \\
\text { plagiados (sin citar). }\end{array}$ & $\begin{array}{l}\text { - Está relacionado } \\
\text { con el tema y los } \\
\text { objetivos; } \\
\text { - Los contenidos } \\
\text { están ordenados } \\
\text { pero puede mejo- } \\
\text { rarse el discurso; } \\
\text { - Los conceptos } \\
\text { principales están } \\
\text { definidos y la } \\
\text { mayoría de afir- } \\
\text { maciones están } \\
\text { justificadas; } \\
\text { - No hay textos de } \\
\text { otros autores sin } \\
\text { citar; } \\
\text { - Pocas fuentes } \\
\text { primarias o fuentes } \\
\text { no actuales }\end{array}$ & $\begin{array}{l}\text { - Está relacionado } \\
\text { el tema y los } \\
\text { objetivos; } \\
\text { - Hay unidad } \\
\text { discursiva dentro } \\
\text { de cada apartado } \\
\text { pero no entre } \\
\text { apartados; } \\
\text { - No hay textos } \\
\text { plagiados; } \\
\text { - El número de } \\
\text { fuentes es adecua- } \\
\text { do para conseguir } \\
\text { los objetivos. } \\
\text { - La mayoría de las } \\
\text { fuentes son rele- } \\
\text { vantes y la mayoría } \\
\text { son primarias. }\end{array}$ & $\begin{array}{l}\text { - Está relacionado } \\
\text { con el problema y } \\
\text { los objetivos; } \\
\text { - Están definidos } \\
\text { todos los concep- } \\
\text { tos y justificadas } \\
\text { todas las afirma- } \\
\text { ciones; } \\
\text { - Los contenidos } \\
\text { dentro y entre } \\
\text { apartados están } \\
\text { enlazados y si- } \\
\text { guen una unidad } \\
\text { discursiva; } \\
\text { - Todas las fuen- } \\
\text { tes son relevantes } \\
\text { y primarias. }\end{array}$ & Nivel 4 & 1,5 & \\
\hline $\begin{array}{l}\text { Marco meto- } \\
\text { dológico o } \\
\text { propuesta de } \\
\text { intervención } \\
(15 \%) \\
-\end{array}$ & $\begin{array}{l}\text { - No es apropiado } \\
\text { para conseguir } \\
\text { los objetivos } \\
\text { formulados } \\
\text { y resolver el } \\
\text { problema; } \\
\text { - No está desa- } \\
\text { rrollado correcta- } \\
\text { mente; } \\
\text { - Resultados } \\
\text { confusos y poco } \\
\text { coherentes (moda- } \\
\text { lidad: iniciación a la } \\
\text { investigación); } \\
\text { - Propuesta o pro- } \\
\text { yecto incompleto } \\
\text { (modalidad pro- } \\
\text { puesta/proyecto). }\end{array}$ & $\begin{array}{l}\text { - Adecuado para } \\
\text { conseguir los } \\
\text { objetivos y resolver } \\
\text { el problema pero } \\
\text { necesita mayor } \\
\text { desarrollo; } \\
\text { - Los resultados son } \\
\text { coherentes con los } \\
\text { objetivos pero falta } \\
\text { descripción (moda- } \\
\text { lidad: iniciación a la } \\
\text { investigación); } \\
\text { - La propuesta o } \\
\text { proyecto incluye } \\
\text { todos los apartados } \\
\text { pero falta desarro- } \\
\text { llo en alguno de } \\
\text { ellos (modalidad } \\
\text { propuesta/pro- } \\
\text { yecto). }\end{array}$ & $\begin{array}{l}\text { - Adecuado } \\
\text { para conseguir } \\
\text { los objetivos } \\
\text { y resolver el } \\
\text { problema; } \\
\text { - Los resultados } \\
\text { son coherentes } \\
\text { con los objetivos, } \\
\text { están bien descri- } \\
\text { tos pero pueden } \\
\text { completarse algo } \\
\text { más (modalidad: } \\
\text { iniciación a la } \\
\text { investigación); } \\
\text { - La propuesta/ } \\
\text { proyecto está } \\
\text { completa y de- } \\
\text { sarrollada pero } \\
\text { puede mejorarse } \\
\text { algún apartado } \\
\text { (modalidad pro- } \\
\text { puesta/proyecto). }\end{array}$ & $\begin{array}{l}\text { - Adecuado } \\
\text { para conseguir } \\
\text { los objetivos } \\
\text { y resolver el } \\
\text { problema; } \\
\text { - Resultados } \\
\text { coherentes, } \\
\text { adecuados y } \\
\text { descritos con de- } \\
\text { talle (modalidad: } \\
\text { iniciación a la } \\
\text { investigación); } \\
\text { - La propuesta/ } \\
\text { proyecto está } \\
\text { completa y se } \\
\text { describe con } \\
\text { detalle (modali- } \\
\text { dad propuesta/ } \\
\text { proyecto). }\end{array}$ & Nivel 4 & 1,5 & \\
\hline $\begin{array}{l}\text { Conclusiones } \\
(10 \%)\end{array}$ & $\begin{array}{l}\text { - No hay } \\
\text { conclusiones. } \\
\text { - Las conclusiones } \\
\text { no responden a los } \\
\text { objetivos plantea- } \\
\text { dos o no son conse- } \\
\text { cuencia del trabajo } \\
\text { desarrollado. }\end{array}$ & $\begin{array}{l}\text { - Hay apartado de } \\
\text { conclusiones pero } \\
\text { no responde a } \\
\text { todos lo objetivos; } \\
\text { - Son consecuencia } \\
\text { del trabajo desarro- } \\
\text { llado pero les falta } \\
\text { originalidad; }\end{array}$ & $\begin{array}{l}\text { - Hay apartado de } \\
\text { conclusiones que } \\
\text { permite conocer si } \\
\text { se han conseguido } \\
\text { los objetivos; } \\
\text { - Son consecuencia } \\
\text { del trabajo desa- } \\
\text { rrollado; } \\
\text { - Son originales } \\
\text { y se expresan } \\
\text { con terminología } \\
\text { propia; }\end{array}$ & $\begin{array}{l}\text { - Hay apartado } \\
\text { de conclusiones } \\
\text { que permite } \\
\text { conocer si se han } \\
\text { conseguido los } \\
\text { objetivos; } \\
\text { - Son consecuen- } \\
\text { cia del trabajo } \\
\text { desarrollado; } \\
\text { - Son originales } \\
\text { y se expresan } \\
\text { con terminología } \\
\text { propia; } \\
\text { - Incluye limita- } \\
\text { ciones y pros- } \\
\text { pectiva (si es } \\
\text { oportuno) }\end{array}$ & Nivel 4 & 1 & 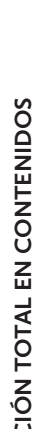 \\
\hline $\begin{array}{l}\text { Referencias } \\
\text { bibliográficas } \\
(10 \%)\end{array}$ & $\begin{array}{l}\text { - No incluye } \\
\text { bibliografía; } \\
\text { - La mayor parte } \\
\text { de las citas en el } \\
\text { texto no aparece } \\
\text { en las referencias } \\
\text { bibliográficas y a la } \\
\text { inversa; } \\
\text { - No hay coherencia } \\
\text { en la forma de } \\
\text { citar en el texto } \\
\text { y tampoco en la } \\
\text { bibliografía. }\end{array}$ & $\begin{array}{l}\text { - Incluye } \\
\text { bibliografía pero } \\
\text { falta alguna de las } \\
\text { referencias citadas } \\
\text { en el texto (máx 3); } \\
\text { - No utiliza nor- } \\
\text { mativa APA pero } \\
\text { la forma de citar } \\
\text { es coherente a lo } \\
\text { largo del docu- } \\
\text { mento. }\end{array}$ & $\begin{array}{l}\text { - Incluye } \\
\text { bibliografía y } \\
\text { todas las citas del } \\
\text { texto se encuen- } \\
\text { tran en la lista de } \\
\text { referencias; } \\
\text { - No utiliza nor- } \\
\text { mativa APA pero } \\
\text { la forma de citar } \\
\text { es coherente a lo } \\
\text { largo del docu- } \\
\text { mento. }\end{array}$ & $\begin{array}{l}\text { - Incluye } \\
\text { bibliografía y } \\
\text { diferencia entre } \\
\text { referencias y } \\
\text { bibliografía } \\
\text { consultada (si es } \\
\text { oportuno); } \\
\text { - Todas las citas } \\
\text { del texto se en- } \\
\text { cuentran la lista } \\
\text { de referencias; } \\
\text { - Utiliza normati- } \\
\text { va APA } \\
\end{array}$ & Nivel 4 & 1 & \\
\hline
\end{tabular}

TABLA N ${ }^{\circ}$ 3. RÚBRICAS PARA LA EVALUACIÓN DE LA MATERIA: INTRODUCCIÓN A LA INVESTIGACIÓN EN ESPACIOS SOCIALES. Fuente: Elaboración Propia 


\section{SERIE VI GEOGRAFÍA}

REVISTA DE LA FACULTAD DE GEOGRAFÍA E HISTORIA
AÑOS 2015-2016

ISSN: $1130-2968$

E-ISSN 2340-146X

\section{8-9 ESPACIO, TIEMPO \\ Y FORMA}

14

PRESENTACIÓN · ForEWORD

\section{Artículos · Articles}

15

Carlos Alberto Abalerón

Diferencias innatas y desigualdades socio-espaciales de Calidad de Vida en San Carlos de Bariloche, Argentina / Innate differences and socio-spatial inequalities Quality of Life in San Carlos de Bariloche, Argentina

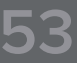

NATACHA CALVET TAPIA

Las prácticas lúdicas en la calle y la imagen del centro de la ciudad en Santiago de Chile / Recreational practices on the street and the image of the city center of Santiago de Chile

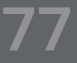

ENRIQUE dE Rosa GIOLITO

Las Complejas relaciones entre paisaje, puerto, ciudad y sus efectos en el patrimonio cultural en la bahía de Pasaia / The complex relationships between landscape, harbor, town and its effects on cultural heritage in the bay of Pasaia

\section{3} ANa María López ORTEgo

¿Quién está contra quién en los cerros orientales de Bogotá?. La perspectiva local desde el barrio la Cecilia / Who is against who in the eastern hills of Bogotá? - the local perspective from the neighborhood of la Cecilia

JULIO LÓPEZ-DAVALILLO LARREA

Las eurociudades rayanas / The ecocities bordering

\section{Beatriz Martínez de Teresa}

Estudio de los recursos hídricos en España: Análisis y caracterización en la Cuenca del Tajo / Study of water resources in Spain:Analysis and characterization in the Tajo Basin

Francisco José Morales YAgo

La incorporación de los portafolios electrónicos en el proceso enseñanza-aprendizaje y evaluación de la geografía en el alumnado de educación a distancia / The incorporation of electronic portfolios learning in geography and evaluation process in distance education students
24. MARcos ORTEga Montequín parado de tres casos en Asturias / Agrological potential in rural land planning; comparative study of three cases in Asturies

269 José Ramón SÁnchez Holgado

Las torres y atalayas de la costa tropical. ¿un recurso turístico pendiente de explotación? / The towers and watchtowers of the Costa Tropical.an Outstanding tourist resource exploitation

287

OSÉ RAMÓn SÁNCHEZ HOLGADO

Inundaciones en la rambla Albuñol. Causas y posibles medidas de intervención / Floods in Albuñol dry riverbed. Causes and possible intervention

\section{Reseñas · Book Review}

319 Yi-fu Tuan. (2015). Geografía romántica. En busca del paisaje sublime. Editado por Biblioteca nueva. Colección: Paisaje y teoría. Edición de Joan Nogué. 177 pp. ISBN: 978-84-16345-15-1 O (AURELIO NiETo CodinA)

323 Bosque Maurel, Joaquín (2011): Granada. Historia y cultura. Granada 323 Diputación Provincial de Granada-Libros de la Estrella, 210 pp. ISBN: 978-84-7807-510-2 (Alejandro García Ferrero)

\section{Imágenes y palabras · Pictures and words}

329 Francisco josé Morales Yago

Las salinas de Imón (Guadalajara): un paisaje cultural de gran valor histórico / The saltworks of Imón (Guadalajara): a cultural landscape of great historical value

\section{Historia de la Geografía Española · History of Geography in Spain}

335

Aurelio Nieto Codina, Dolores Brandis García, Carlos Pardo Abad Tres tesis doctorales dirigidas por don Manuel de Terán; el paisaje residencial en Madrid (Dolores Brandis), la enseñanza en Madrid (Ana Olivera) y el barrio de Salamanca (Rafael Más) / Three thesis directed by don Manuel Terán; residential landscape in Madrid (Dolores Brandis) teaching in Madrid (Ana Olivera) and the Salamanca district (Rafael Más) 